\title{
1D microcavity-based optical parametric oscillator: generation of balanced twin beams in strong and weak coupling regime
}

\author{
Marco Abbarchi, ${ }^{1}$ Vincenzo Ardizzone,${ }^{1}$ Timothee Lecomte, ${ }^{1}$ Aristide Lemaitre, ${ }^{2}$ Isabelle Sagnes, ${ }^{2}$ \\ Pascale Senellart, ${ }^{2}$ Jacqueline Bloch, ${ }^{2}$ Philippe Roussignol, ${ }^{1}$ and Jerome Tignon ${ }^{1}$ \\ ${ }^{1}$ Laboratoire Pierre Aigrain, École Normale Supérieure, CNRS (UMR 8551), \\ Université P. et M. Curie, Université D. Diderot, 75231 Paris Cedex 05, France. \\ ${ }^{2}$ LPN/CNRS, Route de Nozay, F-91460 Marcoussis, France.
}

We report on a detailed experimental investigation of interbranch parametric scattering processes in onedimensional semiconductor microcavities. Band dispersion and corresponding far field emission patterns are studied by polarization resolved and power dependence measurements under resonant and non-resonant excitation at normal incidence. We demonstrate the realization of optical parametric oscillation of balanced twin beams which are degenerate in energy and split in momentum space. This achievement is shown for both the strong and the weak coupling regime highlighting the versatility of this peculiar microcavity system.

PACS numbers: 78.47.1p, 71.36.1c 


\section{I: INTRODUCTION}

Parametric scattering processes (PSPs) and optical parametric oscillation (OPO) have been intensively studied for their numerous applications in non-linear optics. ${ }^{1}$ Parametric amplification, generation of new laser frequencies, twin beams, and photon entanglement are important features of PSPs and OPO. ${ }^{1-3}$ However, poor conversion efficiency, large cavity extension and driving laser power are typical drawbacks of conventional PSPs and OPO. The increasing need of efficient and miniaturized light sources for scalable quantum computation and quantum information protocols ${ }^{4}$ stimulated the recent realization of OPO in semiconductor microcavities (MCs) ${ }^{5}$ toward the realization of electrically-driven quantum emitters in solid state. ${ }^{6}$

Original proposals for the exploitation of parametric phenomena in planar MCs (2D-MCs) were based on the strong lightmatter coupling (SC), which rules the shape of upper and lower polariton branches (UP and LP respectively) dispersions in energy-momentum space. . $^{7,8}$ The excitation at the inflection point of the S-shaped LP branch allows the efficient realization of PSPs with energy and momentum conservation (phase matching conditions, PMCs). ${ }^{7}$ It is now well established that very large $\chi^{3}$ polaritonic non-linearities in 2D-MCs can be used in order to achieve low-threshold OPO between non degenerate signal and idler beams. Nevertheless, this approach suffers from intrinsic drawbacks: (i) the required strong light-matter coupling is usually achieved only at low temperature, (ii) the pump laser must be adjusted to a specific angle, (iii) signal and idler beams are intrinsically strongly unbalanced, thus precluding realistic applications and possible study of quantum correlations.

More recently interbranch parametric scattering in vertical multi-2D-MCs allowed for relevant improvements in the exploitation of 2D-MCs-based OPO: ${ }^{9}, 10$ relaxing the requirement of SC, multi-2D-MCs permit the generation of signal and idler beams at higher operating temperatures in the weak coupling regime (WC). ${ }^{10}$ In this case the twin beams are degenerate in momentum and split in energy (vertical process), preventing an easy spatial separation of the generated signal and idler beams. In addition, with this method, the PMCs impose an equal energy spacing in energy between the three photonic bands and still the extraction efficiency of the idler beam is poor preventing the generation of balanced beams.

In this contribution we show a different approach for the generation of OPO in wire-shaped one-dimensional microcavities (1D-MCs). ${ }^{11}$ The multiplicity of the $1 \mathrm{D}-\mathrm{MC}$ photonic bands ${ }^{12}$ and their fine structures ${ }^{13}$ allow for a versatile engineering of different scattering channels and of twin beams polarization properties. The choice of horizontal scattering processes (signal and idler degenerate in energy and split in momentum space) relaxes the requirements of $\mathrm{SC}^{10,11}$ and does not require an equal spacing of the photonic/polaritonic modes splitting, necessary for achieving vertical OPO. ${ }^{10,13}$ Most importantly, the twin beams generated in horizontal OPO have intrinsically balanced intensity.

We performed polarization resolved measurements both under non-resonant and resonant excitation, at normal incidence. Signal and idler linear polarization may be opposite to that of the excitation laser demonstrating the generation of Rayleigh-free photon beams via polarization inversion mechanism. The onset of a threshold in the power dependence of signal and idler intensities obtained in horizontal processes demonstrates the realization of balanced OPO both at $10 \mathrm{~K}$ in SC as well as $100 \mathrm{~K}$ in the WC.

The investigated sample is grown in a molecular beam epitaxy reactor and is based on a $\lambda / 2$ 2D-MC composed of $\mathrm{Ga}_{0.05} \mathrm{Al}_{0.95} \mathrm{As}^{14}{ }^{14} \mathrm{The}$ cavity is sandwiched between top and bottom Bragg mirrors (respectively 26 and 30 pairs of $\mathrm{Ga}_{0.05} \mathrm{Al}_{0.95} \mathrm{As}_{\mathrm{G}} \mathrm{Ga}_{0.80} \mathrm{Al}_{0.20} \mathrm{As}$ layers). The active medium is constituted of three stacks of four GaAs quantum wells (width $7 \mathrm{~nm}$ ), placed at the antinodes of the cavity mode (one group at the cavity center and the other two at the first antinodes in each Bragg mirror). The layers thickness is wedged, allowing for a fine tuning of the relative energy between the MC photonic mode energy at $\vec{k}=0\left(E_{2 D}\right)$ and the excitonic resonance $\left(E_{X}\right)$. Hereafter $E_{2 D}-E_{X}$ is referred as detuning $\delta$. The 1D-MCs are fabricated by processing the planar 2D-MC by reactive ion etching (the long axis of the wire is set orthogonal to the wedge thus $\delta$ is kept constant along the 1D-MC). Different set of 1D-MCs having width $L_{\perp}$ from 3 $\mu \mathrm{m}$ to $7 \mu \mathrm{m}$ and same length $L_{\|}=1000 \mu \mathrm{m}$ are etched on the same sample. A spacing of $6 \mu \mathrm{m}$ between two adjacent 1D-MCs allows the investigation of individual emitters.

The CW excitation is provided by a tunable Ti:Sapphire laser focused in a long and sharp spot $(\sim 100 \mu \mathrm{m}$ by $\sim 4 \mu \mathrm{m}$ in full width at half maximum) while its polarization is set perpendicular to the wire axis (direction $\perp$ ). The sample is cooled down to cryogenic temperatures in a cold-finger, liquid-helium cryostat allowing for a fine tuning between $5 \mathrm{~K}$ and $300 \mathrm{~K}$.

The far field radiation spectrum of single 1D-MCs is investigated with a method based on the Fourier space imaging (see Fig. 1 a)): the image of the back focal-plane of a micro-objective lens (NA $=0.4$, focal length $f=16 \mathrm{~mm}$ ) is focused on the exit slit of a $50 \mathrm{~cm}$ monochromator and detected by a silicon based CCD camera (the spectral resolution is about $100 \mu \mathrm{eV}$ ).

In our experiment the $1 \mathrm{D}-\mathrm{MC}$ long axis (direction $\|$ ) is set at $\sim 45$ degrees with respect to the entrance slit of the spectrometer (see Fig.1 b)) making possible the simultaneous detection of the band dispersion of even and odd modes which in turn present an intensity maximum or a minimum at $\vec{k}=0$. All the spectra are discriminated in polarization in order to detect fine structure which represents a key feature of our experiment.

Due to the discontinuity in the refractive index in the direction $\perp$, the electric field is also laterally confined. ${ }^{5}$ This discontinuity changes the photonic mode of the original 2D-MC in a set of modes indexed by $j=0,1,2, \ldots$ with energy at $\vec{k}=0$ given by $E_{1 D}^{j}=\sqrt{\left[E_{2 D}\right]^{2}+\left[\left(\hbar c / n_{C}\right)\left(\pi / L_{\perp}\right)(j+1)\right]^{2}}$ where $n_{C}$ is the refractive index of the cavity material (for this reason we refer to the detuning $\delta$ as the energy distance between $\left.E_{X}-E_{1 D}^{j=0}\right){ }^{16,17}$

Fig. 1 c) and d) show colour-intensity maps of typical cavity emission $\left(L_{\perp}=4 \mu \mathrm{m}\right)$ under non resonant excitation and low 
a)

c)
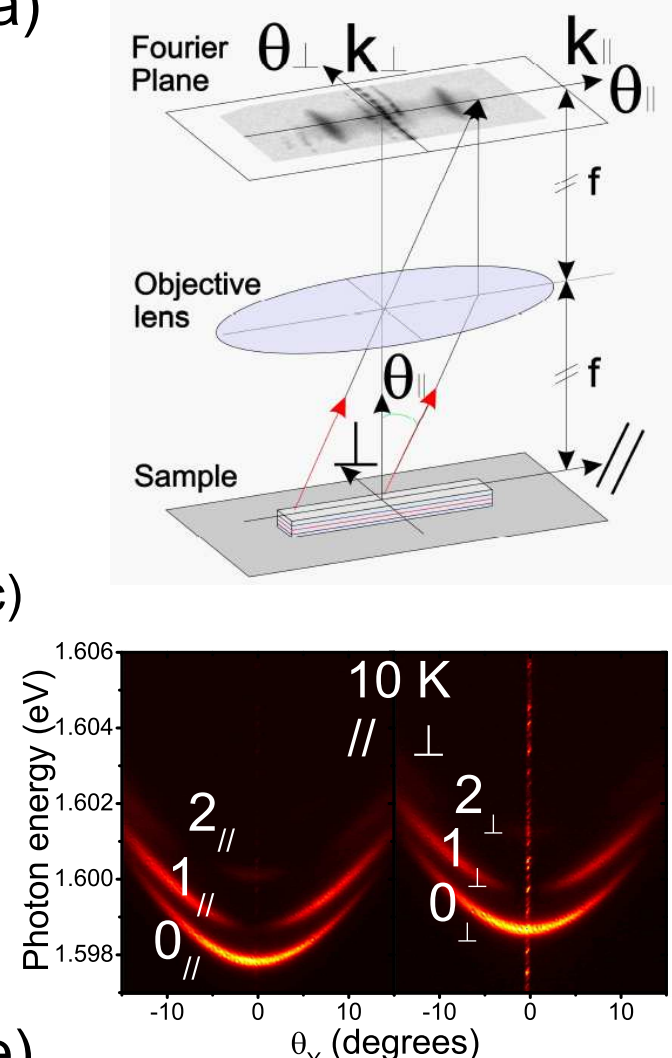

e)

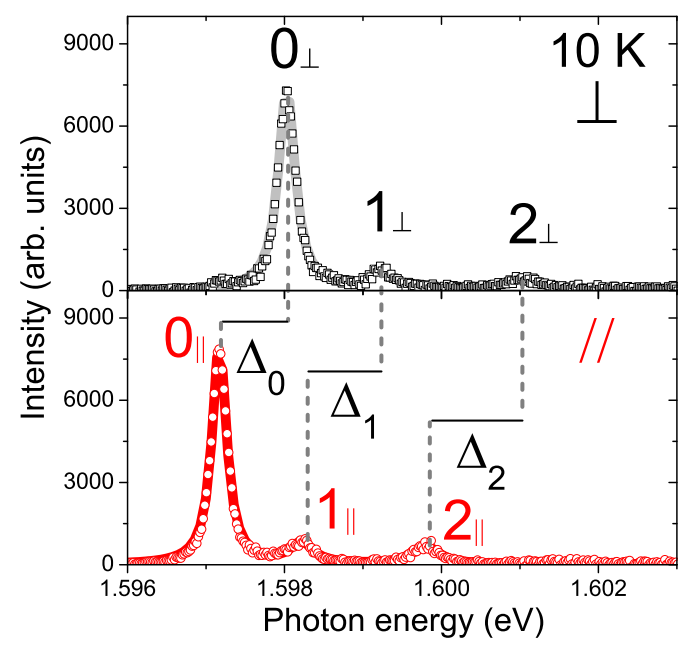

b)

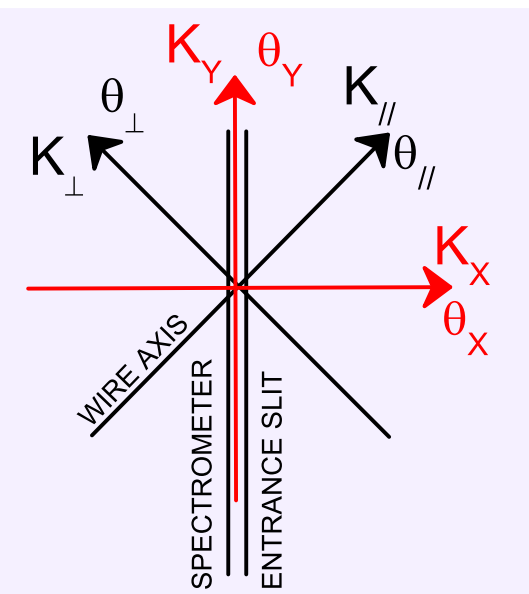

d)

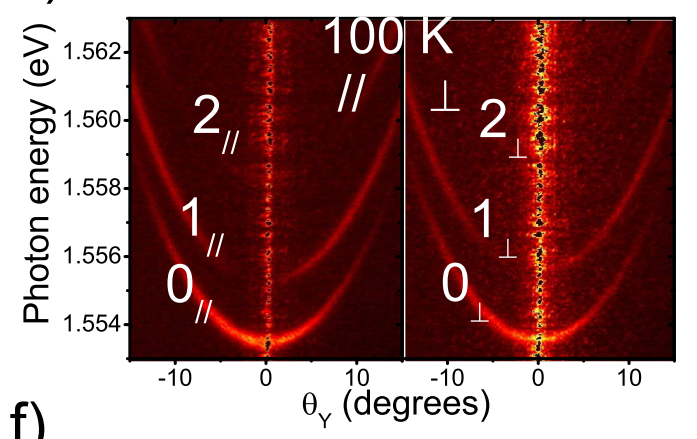

f)

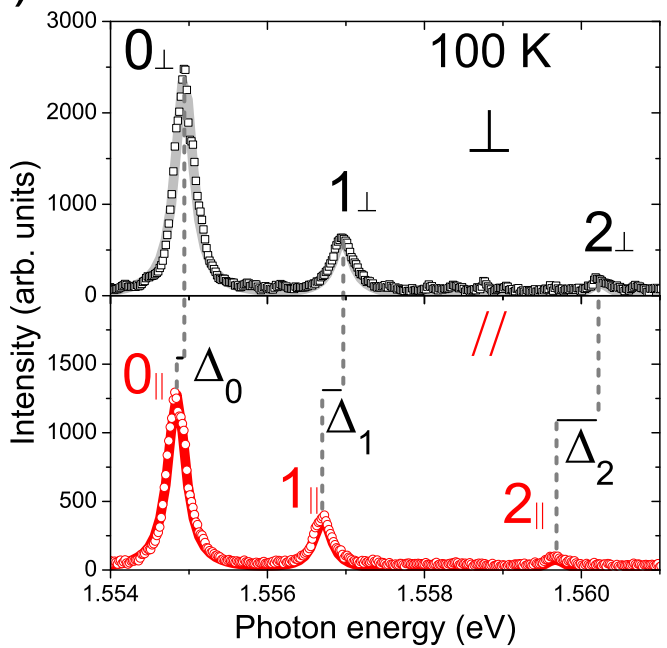

FIG. 1. (Colour online) a) Scheme of the far field imaging technique. b) Scheme of the relative orientation of the different reference frames. c) Colour intensity map of dispersion of a typical 1D-MC at $10 \mathrm{~K}$ in SC regime in energy-momentum space. Left (right) panel reports the $\|$ $(\perp)$ polarization. d) Same as c) at $100 \mathrm{~K}$ in the WC regime for a different cavity. e) Top (bottom) panel: spectra at $\theta_{Y} \simeq 5$ degrees for $\|(\perp)$ polarization at $10 \mathrm{~K}$ as deduced from graph c). f) same as e) at $100 \mathrm{~K}$ as deduced from graph $\mathrm{d}$ )

power $\left(\mathrm{P}_{E x c} \simeq 2 \mathrm{~mW}\right.$ ) at $10 \mathrm{~K}$ and $100 \mathrm{~K}$, respectively (note that the scale of the horizontal axis $\left(\theta_{Y}\right)$ takes into account the relative angle between the 1D-MC axis and the spectrometer entrance slit). The detected polarization in the left (right) panel is $\|(\perp)$ to the $1 \mathrm{D}-\mathrm{MC}$ axis.

At $10 \mathrm{~K}$ the SC is easily achieved by choosing a moderate detunig $(\delta \simeq-5 \mathrm{meV})$. At $100 \mathrm{~K}$ and large detuninig $(\delta \simeq-35$ $\mathrm{meV}$ ) the band dispersions are well approximated by hyperbolas (see Fig. $1 \mathrm{~d}$ )) showing that the observed cavity modes are prevalently photon-like and the system is in WC. However, when reducing $\delta$, we observe strong reduction in the intensity of the 
cavity modes masking all the far field features and preventing an efficient PSP (not shown). In fact at $100 \mathrm{~K}$ the investigation is limited to $\delta \gtrsim 20 \mathrm{meV}$.

Fig. 1 e) and f) shows the two polarized spectra at $\theta_{Y} \simeq 5$ degrees (with this choice we can display the emission of modes with odd $j$ and eventually remove the unwanted laser stray light entering in the spectrometer). As already discussed in Reference 13, polarization fine structures are present: each mode is split in two sub-branches (with linear polarization $\|$ and $\perp$ at lower and higher energy, respectively). The splitting $\left(\Delta_{j}=E_{1 D}^{j}(\perp)-E_{1 D}^{j}(\|)\right)$ was ascribed to mechanical stress due to the different thermal expansion coefficients of the copper cold finger of the cryostat (where the sample was glued at room temperature) and that of the GaAs 1D-MC sample. ${ }^{13}$

At $10 \mathrm{~K}$ and SC the measured value of $\Delta_{j}$ are quite large and increase with $j\left(\Delta_{0}=0.86 \mathrm{meV}, \Delta_{1}=1.02 \mathrm{meV}\right.$ and $\Delta_{2}=1.23$ $\mathrm{meV}$ ). At $100 \mathrm{~K}$ and $\mathrm{WC}$ the polarization splitting is strongly reduced as evidenced in Fig. $1 \mathrm{e}$ ): while $\Delta_{1}$ is still observable, $\Delta_{0}$ is negligible $\left(\Delta_{0}=0.1 \mathrm{meV}, \Delta_{1}=0.26 \mathrm{meV}, \Delta_{2}=0.57 \mathrm{meV}\right)$. In previous reports the large value of $\Delta_{j}$ allowed both to investigate the polarization dynamics of polaritons modes and enabled the realization of vertical OPO via linear polarization inversion mechanism. ${ }^{13}$ Based on the same idea we can obtain balanced twin beams in horizontal OPO as discussed later.

Let's now discuss the resonant excitation regime and the generation of OPO.

Fig. 2 a) shows a colour-intensity map of a 1D-MC dispersion for $\|$ polarization and describes the scheme used for achieving PSPs at $10 \mathrm{~K}$. The value of $\Delta_{0}$, much larger then the line broadening associated to the modes $0_{\perp}$ and $0_{\|}($see Fig. $1 \mathrm{~d})$ ), permits the horizontal PSPs $\left(0_{\perp}, 0_{\perp}\right) \rightarrow\left(0_{\|}, 0_{\|}\right)$by resonantly exciting the $1 \mathrm{D}-\mathrm{MC}$ at the minimum of the $0_{\perp}$ band at $\vec{k}=0$ (this process is schematically depicted on top of Fig 2 a)). The inset in Fig 2 a) shows the corresponding measured process resolved in energy and angle while Fig 2 c) shows the full far field, where the two elongated and bright spots (S and I) are parallel to the laser spot (L) and perpendicular to the wire axis.

At $100 \mathrm{~K}$ the small value of $\Delta_{0}$ (see Fig. $1 \mathrm{~d}$ )) does not allow for an easy separation of S and I from the laser spot whenever exciting on the $j=0$ mode. The small spread in angle of $\mathrm{S}$ and I associated to the process $\left(0_{\perp}, 0_{\perp}\right) \rightarrow\left(0_{\|}, 0_{\|}\right)$, would prevent a clear imaging. As depicted on top of the measured dispersions at $100 \mathrm{~K}$ (see Fig.2 c)) we can exploit the larger splitting $\Delta_{1}$ and resonantly stimulate the process $\left(1_{\perp}, 1_{\perp}\right) \rightarrow(0,0)$ ) by pumping at the minimum of $1_{\perp}$ at $\vec{k}=0$ (in this case both final states in the modes $0_{\perp}$ and $0_{\|}$are possible ${ }^{13}$ ). The inset in Fig. 2 b) and Fig.2 d) show the scattering process resolved in energy-angle space and the corresponding full far field, respectively.

In order to clarify the origin of the measured scattering we perform polarization resolved measurements under resonant excitation which are summarized in Fig. 3. In the top panel is shown the case of PSP at $10 \mathrm{~K}$ where we observe the bright S and I emission at $\theta_{Y}= \pm 7$ degrees only for $\|$ polarization. In particular this demonstrates a complete polarization inversion being the ratio of the intensity in the two modes $\perp / \|$ smaller than $0.5 \%$. In fact, among all the possible PSPs the observed $\left(0_{\perp}, 0_{\perp}\right) \rightarrow\left(0_{\|}, 0_{\|}\right)$is the only allowed by the PMCs. ${ }^{13}$

At $100 \mathrm{~K}$ the picture is quite different: as shown in the bottom panel of Fig. 3 relevant scattering is present for both polarizations $\perp$ and $\|$ at $\theta_{Y}= \pm 10$ degrees (note that the two bands $0_{\perp}$ and $0_{\|}$are largely overlapped both in energy and momentum). This is consistent with the fact that both $\left.\left(1_{\perp}, 1_{\perp}\right) \rightarrow\left(0_{\|}, 0_{\|}\right)\right)$and $\left(1_{\perp}, 1_{\perp}\right) \rightarrow\left(0_{\perp}, 0_{\perp}\right)$ PSPs are allowed by the PMCs. ${ }^{13}$ The relatively large intensity of $\mathrm{S}$ and I for polarization $\|$ demonstrates an efficient polarization inversion mechanism populating the mode $0_{\|}$which intensity is at least the $50 \%$ of that in $0_{\perp}$.

The separation in angle between $\mathrm{S}$ and $\mathrm{I}$ in the process $\left(0_{\perp}, 0_{\perp}\right) \rightarrow\left(0_{\|}, 0_{\|}\right)$at $10 \mathrm{~K}$ is smaller than that in $\left(1_{\perp}, 1_{\perp}\right) \rightarrow(0,0)$ at $100 \mathrm{~K}$. This is consistent with the smaller value of $\Delta_{0}$ with respect to the energy separation $E_{1 D}^{j=1}-E_{1 D}^{j=0}$ which in turn rules the spread in angle of the final states of PSPs at $10 \mathrm{~K}$ and $100 \mathrm{~K}$ respectively.

Further information about the nature of the observed $\mathrm{S}$ and $\mathrm{I}$ in the $0_{\|}$mode can be gathered by power dependence measurements performed in the same resonant conditions previously described. From the full far field images (see Fig. 2 c) and d)) we extract the total intensity of $\mathrm{S}$ and $\mathrm{I}$ as a function of $\mathrm{P}_{E x c}$. The results of this analysis at $10 \mathrm{~K}(100 \mathrm{~K})$ are displayed in left (right) panel of Fig. 4. Both the cases of SC and WC present two distinct regimes: at low $\mathrm{P}_{E x c}$ the detected intensity in the $0_{\|}$mode is dominated by laser scattering and incoherent relaxation (barely linear with $\left.\mathrm{P}_{E x c}\right)$ while above the OPO threshold value $\left(\mathrm{P}_{T h}\right)$ it rapidly increases. By taking into account the dimension of the laser spot on the sample surface we estimate the measured $\mathrm{P}_{T h}$ at $10 \mathrm{~K}$ and $100 \mathrm{~K}$ in $\sim 6 \mathrm{KW} / \mathrm{cm}^{2}$ and $\sim 14 \mathrm{KW} / \mathrm{cm}^{2}$, respectively. More generally the measured values of $\mathrm{P}_{T h}$ range between $\sim 1 \mathrm{KW} / \mathrm{cm}^{2}$ for $\delta \simeq-5 \mathrm{meV}$ up to $\sim 60 \mathrm{KW} / \mathrm{cm}^{2}$ for $\delta \simeq-20 \mathrm{meV}$. This finally demonstrates the generation of OPO of signal (S) and idler (I) beams with equal intensities, as well as relevant spatial separation, in SC and WC.

In conclusion we have shown the realization of OPO in 1D-MCs based on horizontal PSPs. The modes fan of 1D-MCs joined with large linear polarization splitting $\Delta_{j}$ permits an efficient engineering of the parametric scattering channels and allows the generation of OPO both in the SC and in the WC at low and high temperature. The twin beams generated in horizontal processes are intrinsically balanced in intensity and well separated in momentum space. Thanks to the polarization inversion mechanism we can manipulate the polarization of S and I which can be cross-polarized respect to the excitation laser. In the present method, differently from vertical OPO, different interband and sub-band splitting is a tuning tool for setting proper S and I angular spread. Note that the limit of $100 \mathrm{~K}$ for achieving OPO is related to the particular sample in study which was optimized for low temperature investigation. Higher operating temperatures could be in principle reached with appropriate modes energies $E_{1 D}^{j}$, detunings $\delta$ and by tailoring the polarization splitting $\Delta_{j}$. 
a)

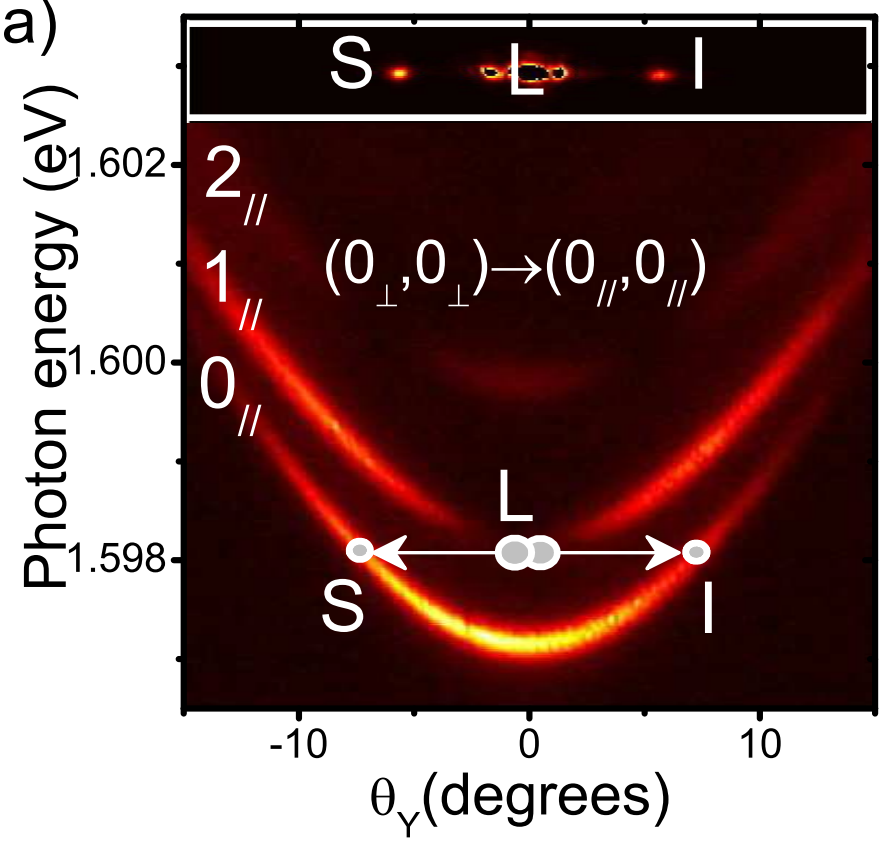

c)

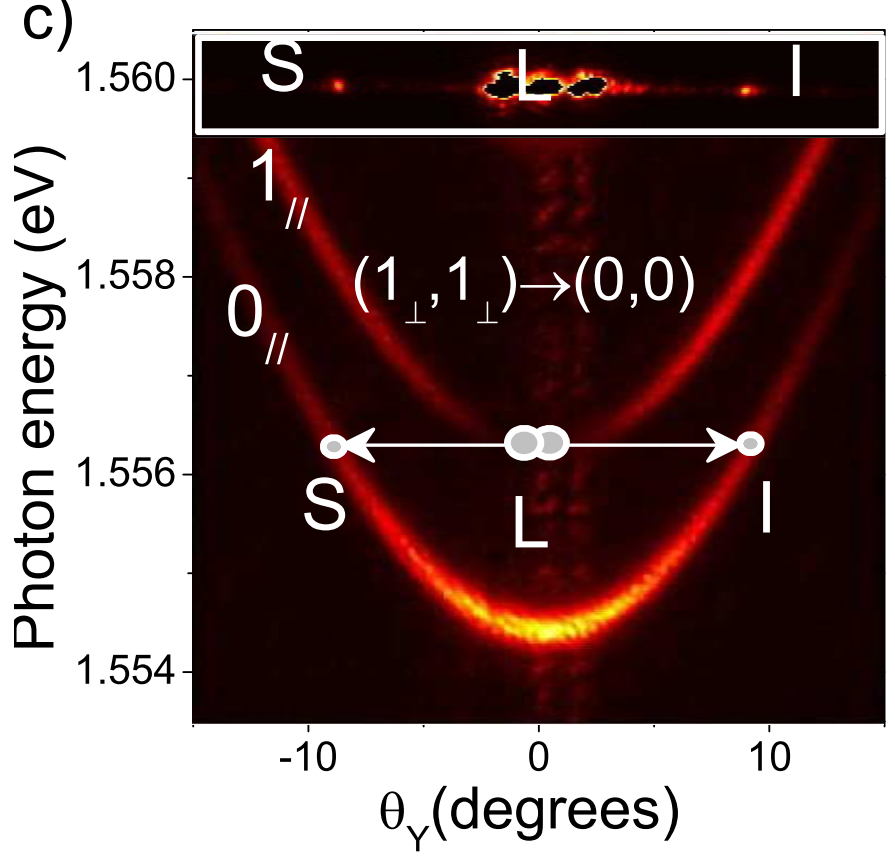

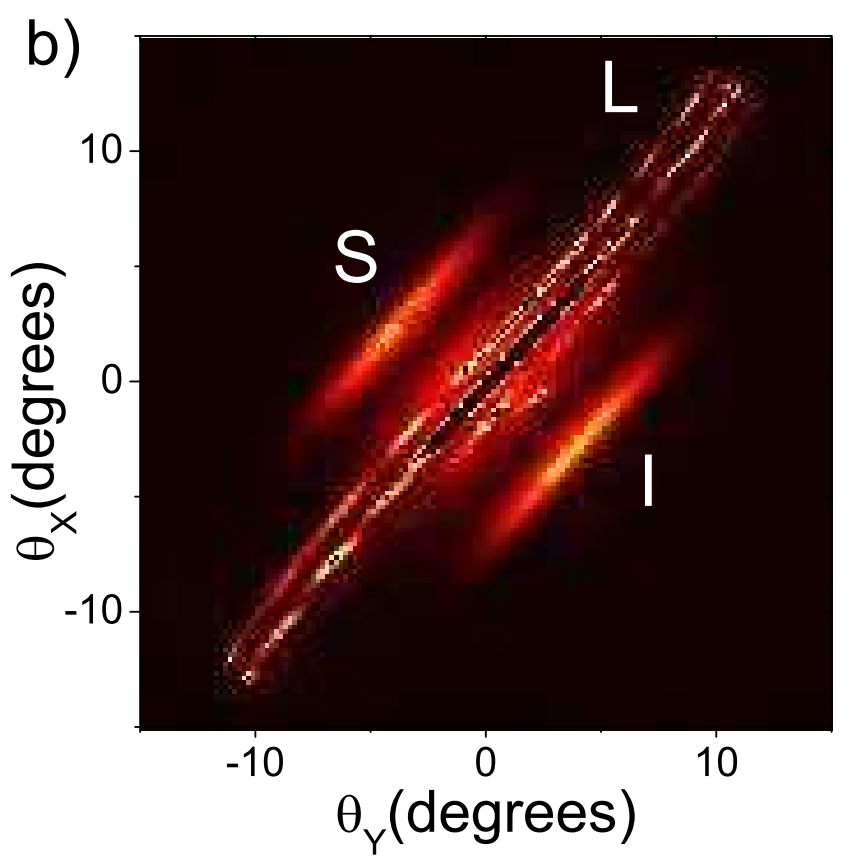

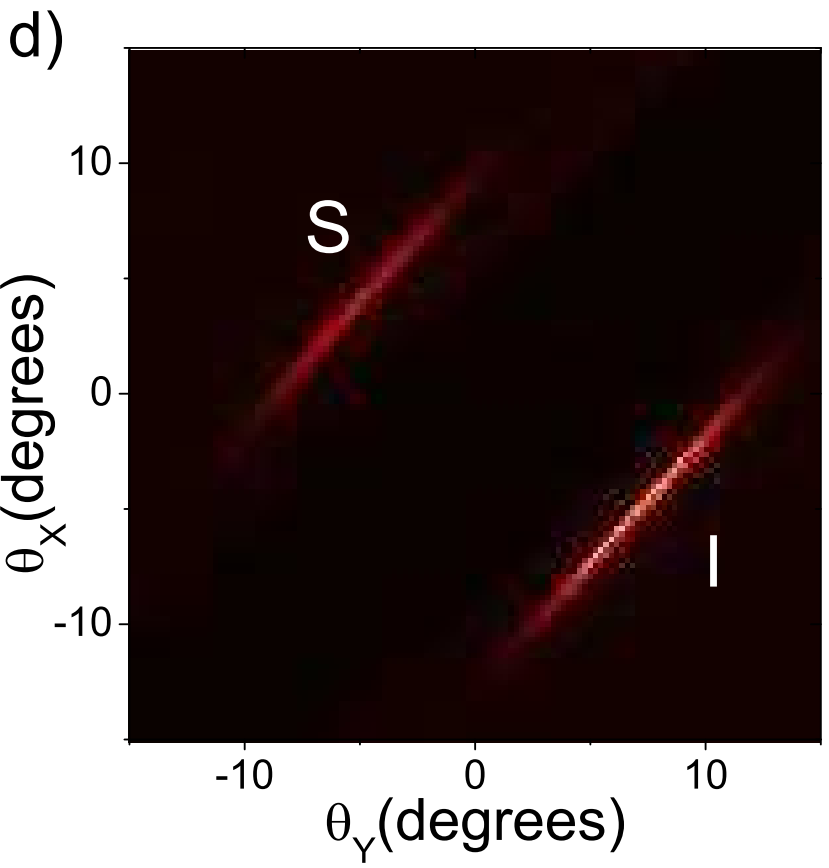

FIG. 2. (Colour online) a) Colour intensity map of a 1D-MC dispersion at $10 \mathrm{~K}$ for $\|$ polarization in energy-momentum space. Dots and arrows indicate the parametric process $\left(0_{\perp}, 0_{\perp}\right) \rightarrow\left(0_{\|}, 0_{\|}\right)$. The inset at top shows the corresponding resonant excitation measurement represented in energy-angle space. b) Full far field associated to $\left(0_{\perp}, 0_{\perp}\right) \rightarrow\left(0_{\|}, 0_{\|}\right)$in angle space at $10 \mathrm{~K}$. c) same as a) at $100 \mathrm{~K}$ for a different cavity. Dots and arrows superimposed indicate the process $\left(1_{\perp}, 1_{\perp}\right) \rightarrow(0,0)$. The inset at top shows the corresponding measured process under resonant excitation represented in energy-angle space. d) Full far field associated to $\left(1_{\perp}, 1_{\perp}\right) \rightarrow(0,0)$ in angle space at $100 \mathrm{~K}$ (in this case the laser was stopped before detection). 


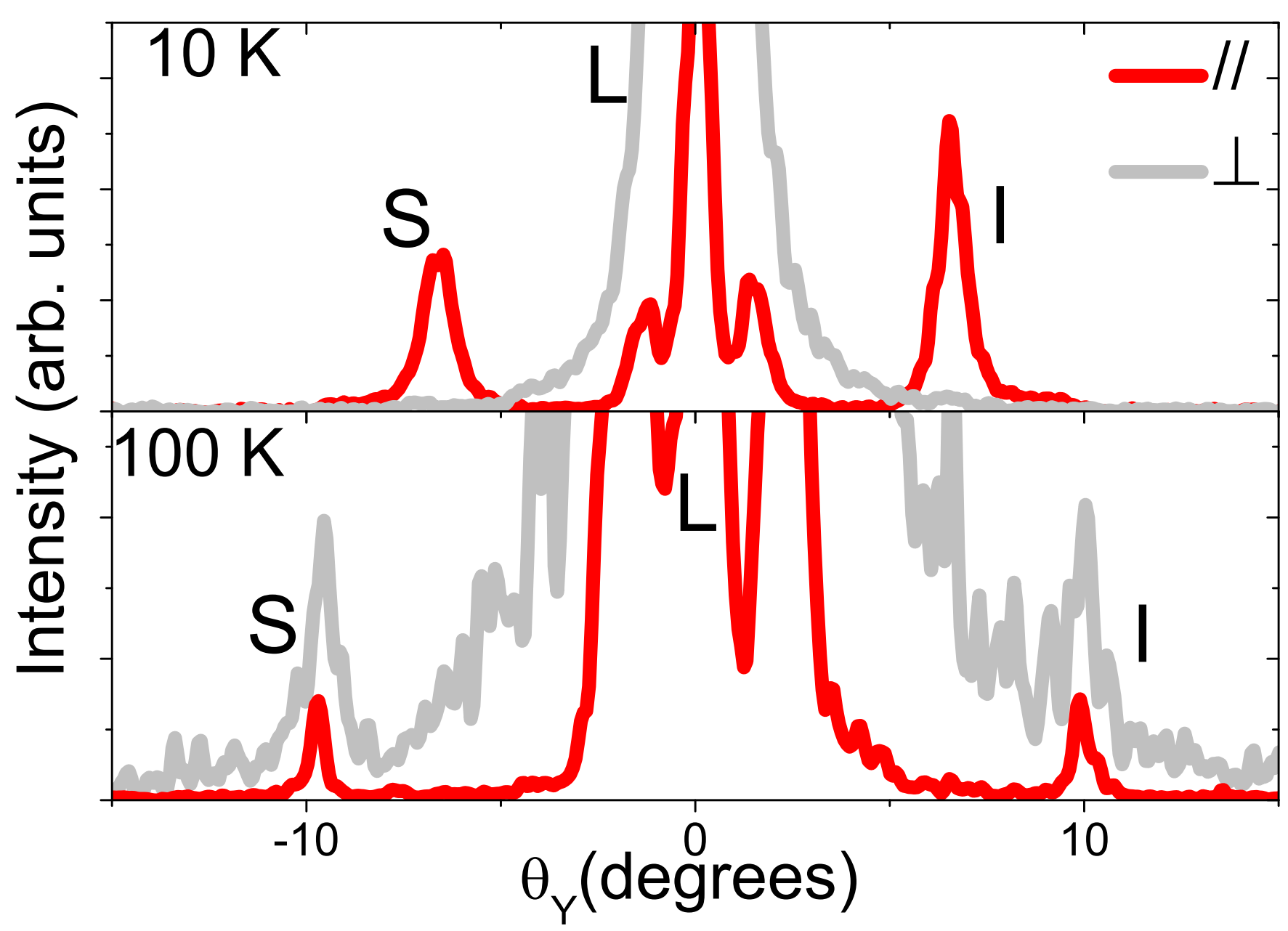

FIG. 3. (Colour online) Top (bottom) panel shows $\|$ and $\perp$ PSPs as a function of $\theta_{Y}$ at $10 \mathrm{~K}(100 \mathrm{~K})$ as deduced by the inset in Fig. 2 a) (Fig. 2 b))

M. A. and V. A. thank the european project EU Network 1IN Clermont-4. 


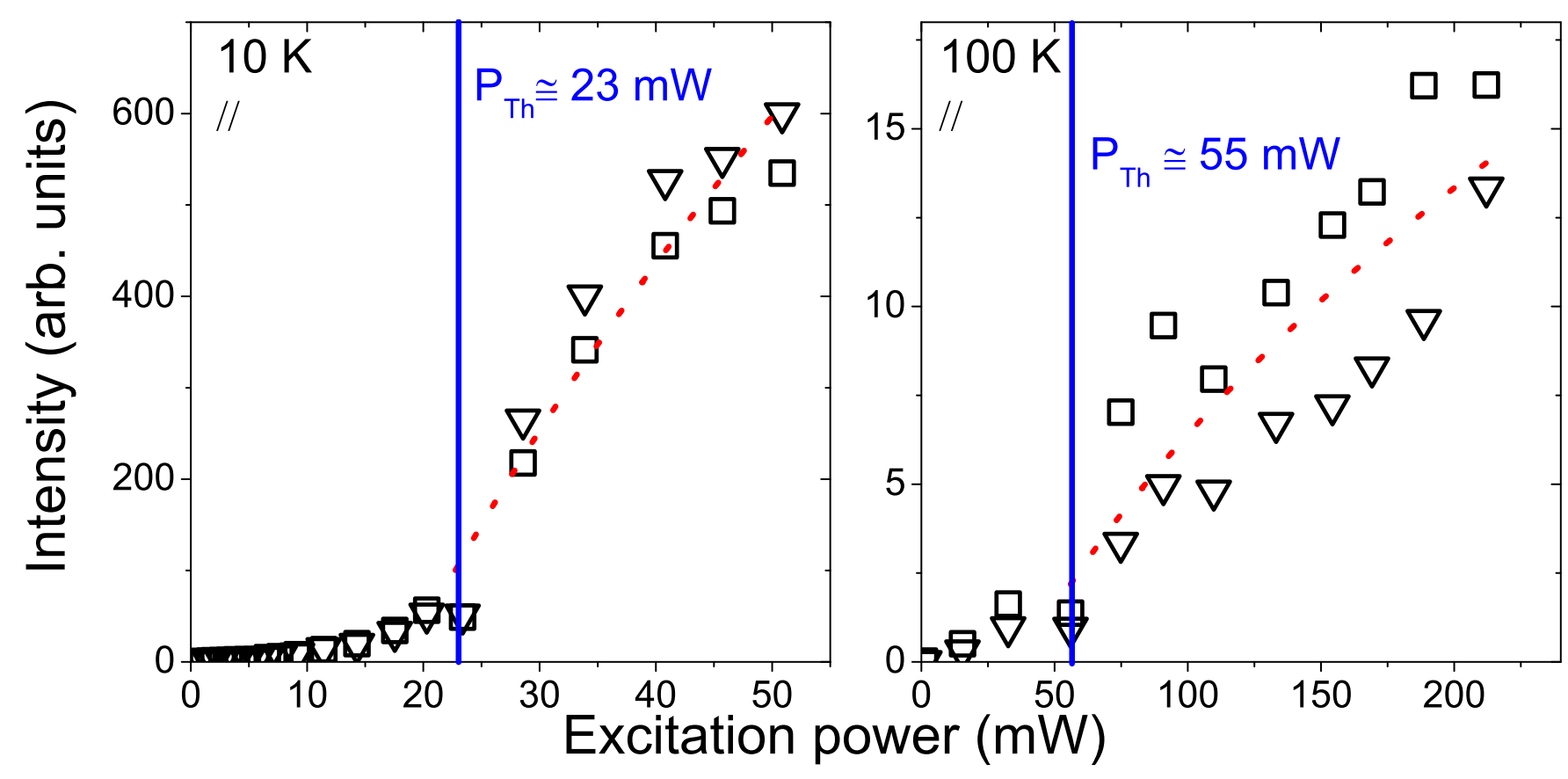

FIG. 4. (Colour online) S and I intensity, respectively full and open symbols, as a function of $P_{E x c}$ at $10 \mathrm{~K}$ (100 K) are shown in left (right) panel. The dashed lines are guides to the eyes and the vertical lines indicate the threshold power $\mathrm{P}_{T h}$. 
1 Y. R. Shen, The Principles of Nonlinear Optics Wiley Inter- Science, Hoboken, (2003)

2 J. A. Armstrong, N. Bloembergen, J. Ducuing, and P. S. Pershan, Phys. Rev. 127, 1918 (1962)

3 K. Edamatsu, G. Oohata, R. Shimizu and T. Itoh Nat. (London) 431, 167 (2004).

${ }^{4}$ D. Bouwmeester, A. K. Ekert, and A. Zeilinger, The Physics of Quantum Information: Quantum Cryptography, Quantum Teleportation, QUantum Computation (Springer-Verlag, Berlin, 2000)

5 A. V. Kavokin, J. J. Baumberg, G. Malpuech, and F. P. Laussy, Microcavities (Oxford University Press, New York, 2007)

${ }^{6}$ J. U. Fürst, D.V. Strekalov, D. Elser, A. Aiello, U. L. Andersen, Ch. Marquardt and G. Leuchs Phys. Rev. Lett. 106, 113901 (2011)

7 P. G. Savvidis, J. J. Baumberg, R. M. Stevenson, M. S. Skolnick, D. M. Whittaker, and J. S. Roberts, Phys. Rev. Lett. 84, 1547 (2000)

${ }^{8}$ M. Saba, C. Ciuti, J. Bloch, J. V. Thierry-Mieg, R. Andre, L. S. Dang, S. Kundermann, A. Mura, G. Bongiovanni, J. L. Staehli and B. Deveaud, Nat. 414, 731 (2003)

9 C. Diederichs and J. Tignon, Appl. Phys. Lett. 87, 251107 (2005)

${ }^{10}$ C. Diederichs, J. Tignon, G. Dasbach, C. Ciuti, A. Lematre, J. Bloch, Ph. Roussignol, and C. Delalande, Nature London 440, 904 (2006)

11 D. Taj, T. Lecomte, C. Diederichs, Ph. Roussignol, C. Delalande, and J. Tignon Phys. Rev. B 80, 081308R (2009)

${ }^{12}$ G. Dasbach, M. Schwab, M. Bayer, D.N. Krizhanovskii, and A. Forchel, Phys. Rev. B 71, 201201R (2002)

13 G. Dasbach, C. Diederichs, J. Tignon, C. Ciuti, Ph. Roussignol, C. Delalande, M. Bayer, and A. Forchel, Phys. Rev. B 71, 161308R (2005)

${ }^{14}$ E. Wertz, L. Ferrier, D. D. Solnyshkov, P. Senellart, D. Bajoni, A. Miard, A Lemaitre, G. Malpuech, and J. Bloch, Appl. Phys. Lett. 95, 051108 (2009)

15 E. Wertz, L. Ferrier, D. Solnyshkov, R. Johne, D. Sanvitto, A. Lemaitre, I. Sagnes, R. Grousson, A. V. Kavokin, P. Senellart, G. Malpuech and J. Bloch, Nature Physics, 6, 860 (2010).

16 C. Ciuti, Phys. Rev. B 69, 245304 (2004)

17 A. I. Tartakovskii, V. D. Kulakovskii, A. Forchel, and J. P. Reithmaier, Phys. Rev. B 57, R6807 (1998) 